\title{
Un análisis crítico sobre el uso de macroalgas como base para una acuicultura sustentable
}

Critical analysis on the use of macroalgae as a base for sustainable aquaculture

\author{
ALEJANDRO H. BUSCHMANN *, ROBERT A. STEAD, MARÍA C. HERNÁNDEZ-GONZÁLEZ, SANDRA V. PEREDA, \\ JAVIER E. PAREDES \& MIGUEL A. MALDONADO
}

Centro i-mar, Universidad de Los Lagos, Camino Chinquihue $\mathrm{km}$ 6, Puerto Montt, Chile

*Autor correspondiente: abuschma@ulagos.cl

\begin{abstract}
RESUMEN
El presente trabajo analiza tanto situaciones ambientales y de mercado como las regulaciones legales que actualmente limitan el desarrollo de la acuicultura de algas en Chile y se discute sobre posibles beneficios e incertidumbres ambientales frente al desarrollo de esta actividad. Chile posee condiciones ambientales extremadamente favorables para fomentar actividades acuícolas, validez demostrada por la exitosa producción de salmonídeos y mejillones así como de otras especies de invertebrados como abalones, ostiones y del alga roja Gracilaria chilensis. Este trabajo señala que, sin embargo, esta oportunidad de negocio y desarrollo de la acuicultura no puede sustentarse en desmedro del patrimonio ambiental del país. Con el fin de balancear el flujo de materia y energía en sistemas costeros utilizados por prácticas de acuicultura, la diversificación acuícola debe estar basada en la incorporación de especies con funciones ecológicas diferentes (productores primarios, detritívoros, herbívoros y carnívoros), lo cual hoy se conoce como Acuicultura Integrada Multi-Trófica (IMTA). Si Chile aborda este tema en profundidad, no solo podría ser un exportador de nuevos productos alimentarios, sino además de tecnologías de producción sustentable para la acuicultura mundial.
\end{abstract}

Palabras clave: acuicultura integrada multi-trófica, acuicultura sustentable, impacto ambiental, regulaciones ambientales para la acuicultura.

\begin{abstract}
The current paper analyses the market, environmental and regulation issues limiting the development of alga aquaculture in Chile and we discuss the possible benefits and uncertainties for the future development of this activity. In particular, Chile has extremely favorable environmental conditions for aquaculture, which allowed that during the past decades the development of a salmon and mussel aquaculture industry and as well as activities with invertebrate species such as abalones and scallops and the red alga Gracilaria chilensis. The benefits and environmental uncertainties for such development are discussed. Chile has extremely favorable environmental conditions for the development of aquaculture, thus we discussed how this business cannot be developed affecting negatively the environmental patrimony of the country. We state that diversification in aquaculture must be based on species with different ecological functions (i.e. primary producers, herbivores, carnivores, and decomposers), which is currently known as Integrated Multi-Trophic Aquaculture (IMTA). This can allow for the balance in energy and matter in coastal systems used in aquaculture. Chile has the opportunity to address these issues in depth, which can allow the country not only to export food products, but also to be a leader in technology for a sustainable aquaculture production worldwide.
\end{abstract}

Key words: environmental impact, environmental regulations for aquaculture, IMTA, integrated multi-trophic aquaculture, sustainable aquaculture.

\section{INTRODUCCIÓN}

El desarrollo de la acuicultura en el mundo está creciendo sostenidamente como alternativa al uso de recursos pesqueros que son cada vez más escasos (Duarte et al. 2007). Con el fin de alcanzar los requerimientos pesqueros mundiales, el desarrollo de la acuicultura está realizando esfuerzos tecnológicos y productivos para expandirse hacia zonas geográficas climáticamente más extremas (hacia latitudes altas) e incluso en condiciones 
de mar abierto (e.g., Troell et al. 2009). Por otro lado, actualmente, la acuicultura está bajo estricto escrutinio público con el fin de compatibilizar el desarrollo económico/social con la conservación del patrimonio ambiental. Así, el creciente interés sobre el potencial acuícola en el mundo y su expansión, está generando cuestionamientos ambientales tales como el incremento en la demanda de recursos (principalmente para producción de harina y aceite de pescado) (Naylor et al. 2001, Olsen 2011), así como por los diversos efectos ambientales generados por desechos inorgánicos tales como acumulación de cobre en el fondo marino (Haya el al. 2001 y Buschmann \& Fortt 2005), aumento de materia orgánica y disminución de oxígeno en los fondos, bajo balsas jaula (Hargrave 2010 , Soto \& Norambuena 2004) y efectos biológicos tales como el aumento de parásitos (Carvajal et al. 1998, Sepúlveda et al. 2004) y el escape de peces en cultivo que conlleva efectos negativos en la fauna nativa (e.g. Soto et al. 2001, 2006, Becker et al. 2007). En Chile estos aspectos también han estado en el centro de la discusión sobre la relación acuicultura y ambiente (ver Buschmann et al. 2006a), en busca de alternativas que permitan reducir potenciales externalidades ambientales adversas (Buschmann et al. 2009).

Sobre la eficiencia del uso del ambiente destinado a acuicultura, se ha estimado el área que requiere esta actividad para obtener todos los recursos necesarios para mantener su ciclo productivo y además asimilar sus desechos -huella ecológica. La superficie calculada para estos fines van desde 4 - 5 hasta valores tan altos como 50000 hectáreas por cada hectárea de cultivo (Folke et al. 1998). Esta variabilidad depende principalmente de los métodos de cultivo aplicados y de las especies cultivadas. En general organismos carnívoros tienen una huella ecológica mayor, en uno o dos órdenes de magnitud, que organismos extractivos (filtradores y algas) que no requieren de un suplemento energético exógeno al medio ambiente (Folke \& Kautsky 1989, Folke et al. 1998). A partir de ello se ha planteado la necesidad de instalar, en sitios de acuicultura, sistemas donde diferentes tipos de organismos reciclen los desechos vertidos al medio, permitiendo la remoción de estos elementos del ecosistema (Troell et al. 1999, Buschmann et al. 2008a, Chopin et al. 2008). A estos sistemas de acuicultura integrada, que utilizan múltiples especies de diferentes niveles tróficos, hoy se les conoce con el nombre de Acuicultura Integrada Multi-trófica (sigla en inglés IMTA) (Chopin et al. 2001). No obstante, como se mencionará más adelante en este trabajo, es necesario evaluar críticamente el por qué estos sistemas no se han desarrollado en Chile pese al gran desarrollo que la acuicultura ha tenido en las últimas décadas (Buschmann et al. 2009).

En este trabajo planteamos, dentro de este contexto ambiental, la función que el cultivo de algas, como organismos extractores de elementos inorgánicos (carbono, fósforo y nitrógeno) puede tener para la salud de los ecosistemas costeros. Actualmente ciertos procesos de eutrofización costera se relacionan con la aparición de florecimientos algales, tanto de micro como macroalgas, siendo esto uno de los grandes problemas ambientales que afectan a zonas costeras (Clarke et al. 2006, Conley et al. 2009). Estos procesos de eutrofización están directamente asociados a emisiones de elementos inorgánicos como son los compuestos nitrogenados (nitratos y amonio). Estos compuestos proceden de efluentes urbanos, actividades agrícolas y deforestación que a través de cuerpos de agua dulce llegan a zonas costeras, así como de la propia actividad acuícola (Anderson et al. 2002, Buschmann et al. 2006a, Liu et al. 2013). En este trabajo se presenta un análisis sobre el desarrollo acuícola tanto globalmente como en Chile, y se discute las ventajas y restricciones que supone la incorporación de algas para propiciar una acuicultura que tienda a ser más sustentable, minimizando los efectos de exceso de nutrientes inorgánicos en zonas costeras y que además puedan generar nuevos negocios. Otros estudios previamente publicados exponen detalladamente los avances y desafíos que el cultivo de algas tiene en Chile y por ello este eje de discusión no está abordado en este trabajo (ver revisión Buschmann et al. 2008b).

\section{UNA VISIÓN SOBRE EL DESARROLLO DE LA ACUICULTURA EN CHILE}

La acuicultura marina aumentó en Chile desde una producción de 361000 toneladas totales en 1998 hasta una producción superior a las 870000 toneladas durante el 2010. Ello posicionó 
mundialmente a este país entre los 10 mayores productores, y como primer productor del mundo occidental. En el mismo período, la salmonicultura se transformó en el principal desarrollo acuícola en Chile, llegando en 2006 a producir 630647 toneladas, descendiendo posteriormente (Fig. 1A) a consecuencia del virus ISA (Godoy et al. 2008).

La producción de moluscos (principalmente abalones, mejillones, ostiones y ostras) alcanzó en el 2010 las 212210 toneladas, siendo los choritos o mejillones el principal producto (Fig. 1B). Las algas producidas en cultivo no
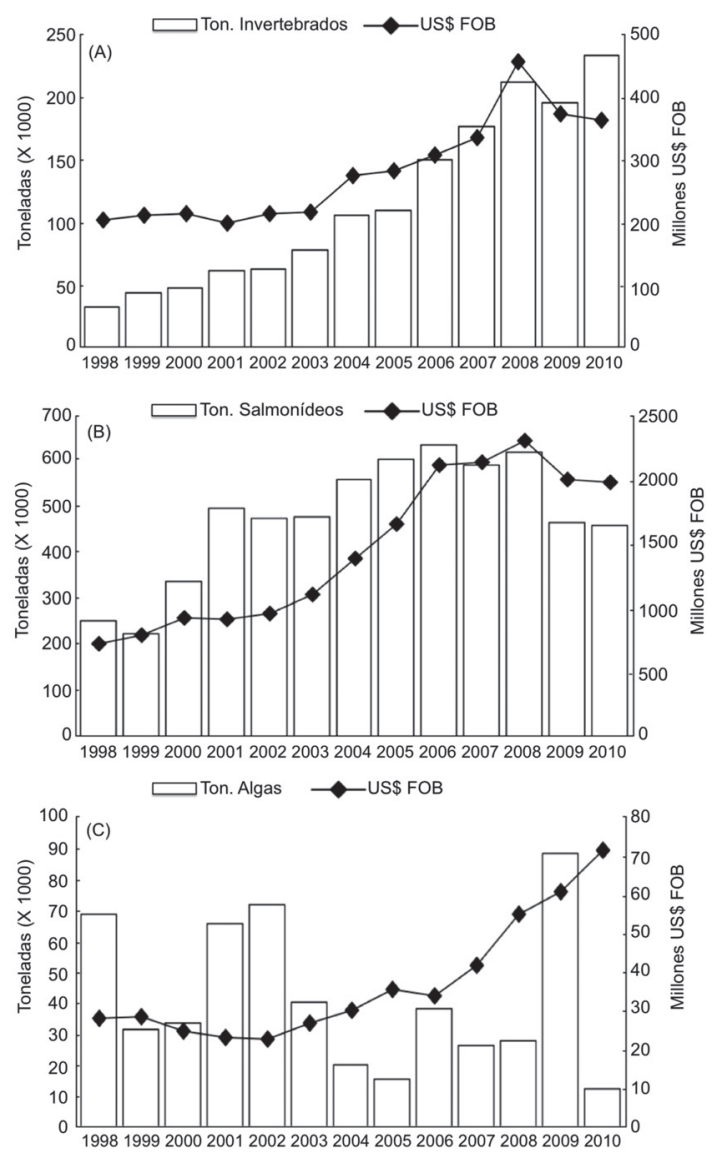

Fig. 1: Producción bruta (toneladas) y valor fob de exportación (millones de US\$̦) en Chile de cultivo de salmones (A), moluscos (B) y algas (C) (fuente: Sernapesca 2012). FOB = Franco a bordo.

Gross aquaculture production (Tons) and fob exportation values (Millions of US\$) of salmon (A), molluscs (B) and algae (C) aquaculture in Chile (source: Sernapesca 2012). $\mathrm{FOB}=$ free on board han mostrado un incremento significativo, con un promedio de 58740 toneladas durante los últimos 10 años (Fig. 1C). No obstante, la extracción y desembarque total de algas en Chile superó las 380000 toneladas el 2010 (ver anuarios de Sernapesca 2012), cifra que destaca el potencial productivo que las algas tienen en la costa de Chile. En particular el cultivo del alga Gracilaria chilensis Bird, McLachlan \& Oliveira sigue predominando en los desembarques por concepto de acuicultura (> 90\% de la biomasa), pero hoy existen las bases tecnológicas para iniciar actividades de acuicultura de varias especies de algas pardas (e.g., Macrocystis pyrifera (L.) C. Ag.) y algas rojas (Sarcothalia crispata (Bory) Leister, Gigartina skottsbergii Setchell \& Gardner, Chondracanthus chamissoii (C. Ag.) Kützing) (ver revisiones de Santelices 1996, Buschmann et al. 2001, 2008b).

Estos aumentos de producción han ido acompañados con un uso cada vez más intensivo de las zonas costeras (Fig. 2) con una expansión de la acuicultura desde la Región de los Lagos hacia la Región de Aysén (ver mapa en Buschmann et al. 2006a). Aunque en muchas situaciones el cultivo de mejillones es considerado extensivo o de pequeña escala según la legislación vigente, es evidente que, debido al aumento de la actividad tanto en número de centros como en las cargas de biomasa en ciertas zonas del sur de Chile, dejó de ser una actividad de pequeña escala desde el punto de vista ambiental. El costo ambiental, solo de la salmonicultura, alcanza al menos el $30 \%$ del producto interno bruto (PIB) de la actividad pesquera en Chile (Buschmann \& Pizarro 2001). Dicho costo se asocia principalmente al cultivo de especies de alto nivel trófico (carnívoros) que requieren de una fuente de energía exógena (alimento) y produce, por tanto, el ingreso de grandes concentraciones de nutrientes, incluso en situaciones de alta eficiencia de conversión del alimento. Se ha determinado que la recuperación de los desechos (nitrógeno, fósforo y carbono), a través de la cosecha del organismo en cultivo, no supera el $75 \%$ (ver a modo de ejemplo balance de masa en Buschmann et al. 1996a). Aunque la eficiencia en el uso de nitrógeno es comparativamente mayor en salmones que en vacunos, la actividad salmonera de la región de Los Lagos aporta 


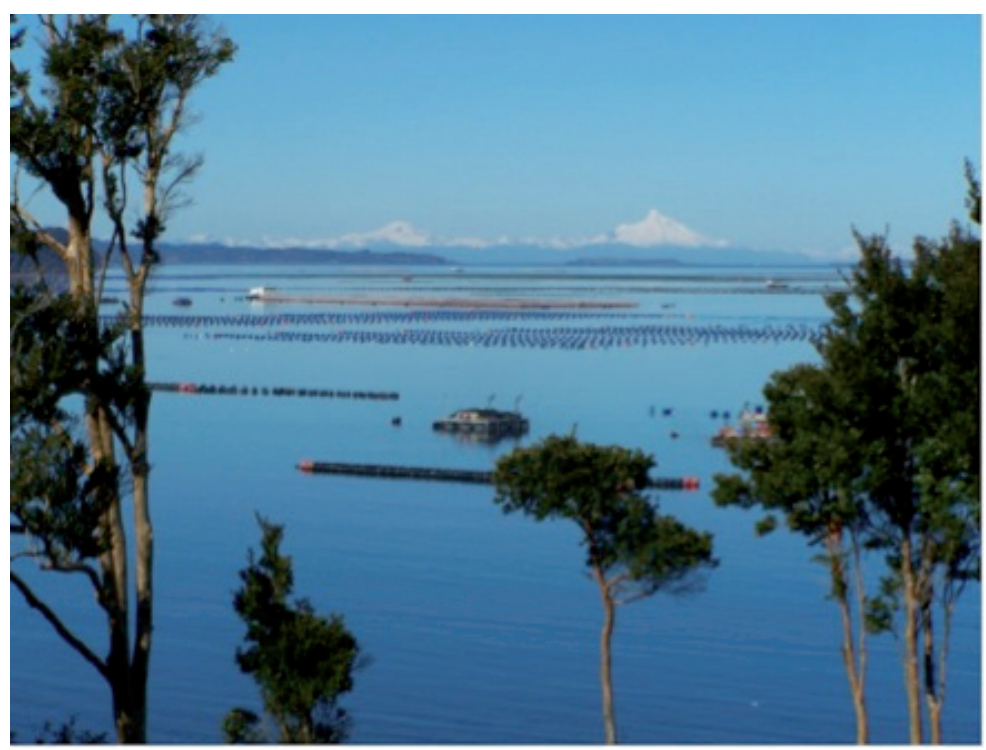

Fig. 2: Fotografía de zona costera de Chiloé en la cual se muestra el grado de uso intensivo que la acuicultura de salmones y mejillones tiene actualmente en sur de Chile.

A picture of the Chiloé coastal area showing the actual state that intensive salmon and mussel aquaculture has in southern Chile.

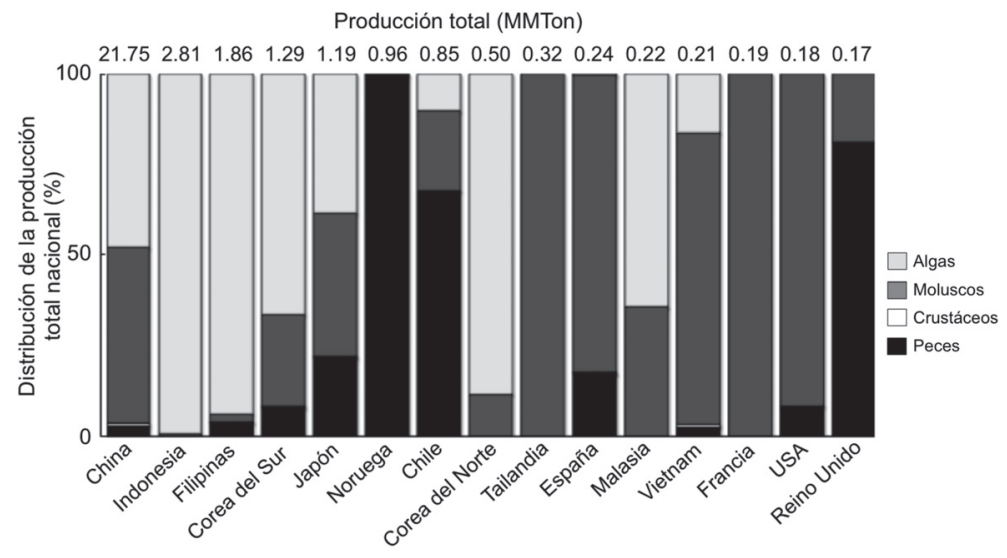

Fig. 3: Gráfico que muestra la producción en millones de toneladas métricas (números sobre barras) y la proporción porcentual de la producción de algas, moluscos, crustáceos y peces en los 15 principales productores del mundo para el año 2010 (fuente: FAO 2010).

Graph showing production in million tons (numbers on bars) and the percent proportion of algae, molluscs, crustaceans and fish production in the main 15 larger worldwide producers for 2010 (Source: FAO 2010).

más nitrógeno a los sistemas acuáticos de agua dulce y marinos que lo que aporta la ganadería de la zona (Soto et al. 2007). Vemos, por tanto, cómo diversos y complejos efectos ambientales se relacionan con la actividad, donde gran variedad de desechos orgánicos e inorgánicos son introducidos a sistemas acuáticos (ver mayores detalles en Buschmann et al. 2009). Si bien Chile es uno de los mayores productores en acuicultura marina, existe una gran diferencia con respecto a la producción acuícola de países orientales, dado que somos un país productor, principalmente, de organismos carnívoros (Fig. 3). Evidentemente 
la gran actividad acuícola que sustenta a países como Japón y China debe basarse en modelos que permitan balancear desechos orgánicos e inorgánicos generados por ciertos organismos, con aquellos otros que permiten reciclarlos y que, al ser cosechados, facilitan su extracción del medio ambiente (Shi et al. 2011). Así el concepto de Acuicultura Integrada Multi-Trófica adquiere especial relevancia como herramienta para reducir los riesgos ambientales, en este contexto las algas adquieren una función preponderante en la reducción de nutrientes inorgánicos disueltos (Chopin et al. 2008, 2011, Buschmann et al. 2008a).

Diversos efectos producidos por la acuicultura propician distintas situaciones ambientales, desfavorables incluso para la misma actividad. Estas deben ser identificadas para poder generar soluciones concretas y apropiadas. Tal es el caso, por ejemplo, de la introducción de nitrógeno en zonas costeras, cuya alta concentración en el mar puede inducir procesos de eutrofización que favorece la proliferación de algas verdes (mareas verdes) tal y como sucedió en Beijing durante las pre-olimpiadas (Liu et al. 2013). En Chile, esta asociación causa-efecto no ha sido comprobada, pero se observan proliferaciones de algas verdes en bahías con acuicultura intensiva y sobrecrecimiento de estas sobre otros organismos costeros (ver Fig. 4). La descomposición de estas proliferaciones de algas aumenta la demanda de oxígeno y por consiguiente pérdidas de biodiversidad, pudiendo incluso disminuir el potencial productivo de las actividades acuícolas (Peckol \& Rivers 1996). Muchos centros de cultivos de salmones tienen problemas de oxígeno en ciertas estaciones del año. Este fenómeno puede no producir mortalidad directamente, pero puede inducir condiciones de estrés tales que provoquen una disminución de las respuestas inmunológicas de los peces, incrementando sus patologías y llegando a alcanzar mortalidades de hasta un 50 \% (Bravo \& Midtlyng 2007, Bustos et al. 2011).

Para poder resolver la problemática ambiental descrita, no basta con incrementar el uso de fármacos como control de patógenos y parásitos, sino además comprender cómo los organismos en cultivo interactúan con el medio ambiente. El excesivo uso de antibióticos aplicados en acuicultura en Chile
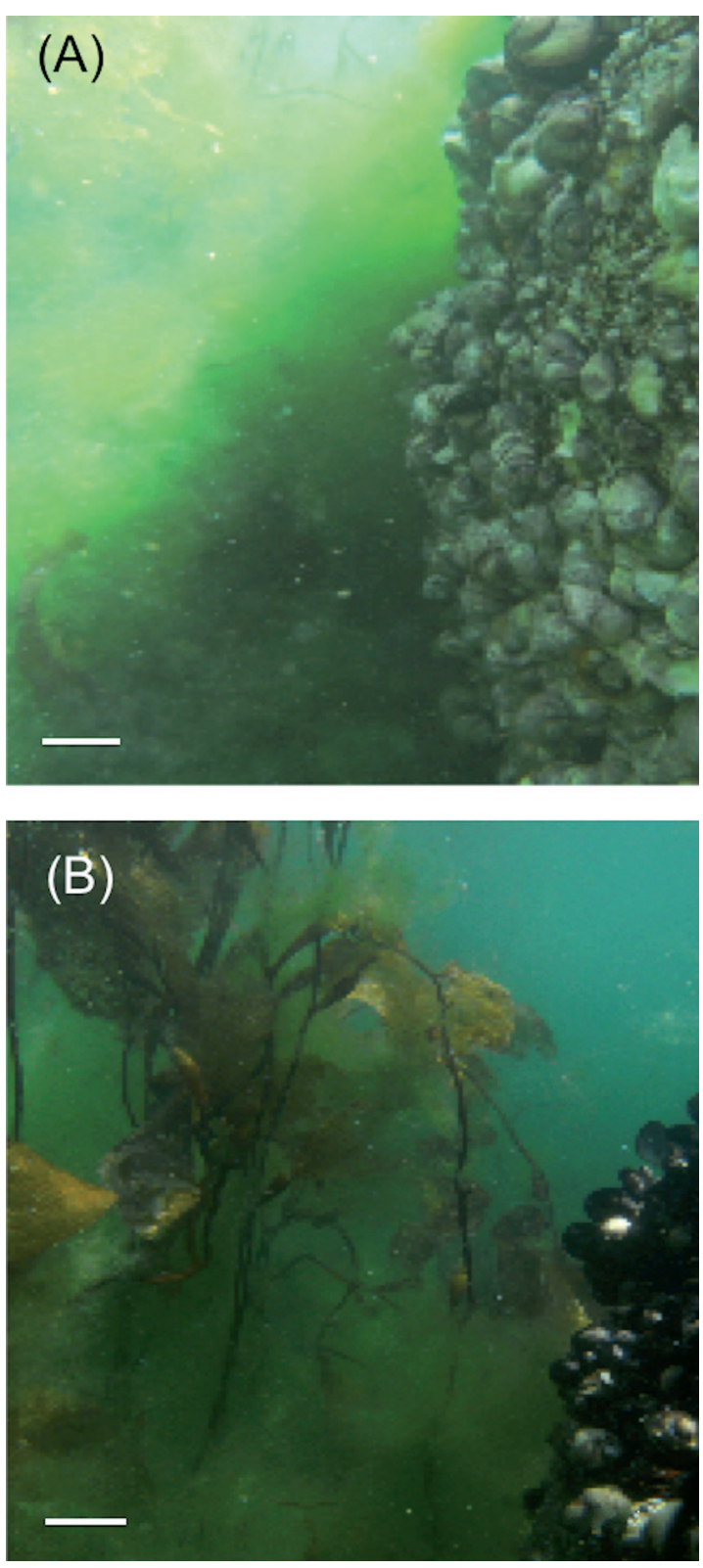

Fig. 4: Fotografías submarinas tomadas en cercanías a sistema de cultivo de salmones y mejillones en la zona de Calbuco. Se observa el desarrollo de algas verdes filamentosas depositándose en el fondo (A) y sobrecreciendo al alga parda Macrocystis pyrifera (B). Barra $=15 \mathrm{~cm}$.

Submarine pictures taken near salmon and mussel farms in Calbuco, Chile showing the development of green algal filaments growing in the bottom (A) and overgrowing the brown algae Macrocystis pyrifera (B). Scale bar $=15 \mathrm{~cm}$.

está relacionado con el tipo y frecuencia de enfermedades, pero su uso en altas dosis puede afectar también a poblaciones 
naturales de organismos costeros (Fortt et al. 2007), e incrementar en ellos, algunos de consumo humano, la resistencia frente a antibióticos (Chelossi et al. 2003, Cabello 2006, Nonaka et al. 2007, Heuer et al. 2009, Buschmann et al. 2012). Desencadenar respuestas epidemiológicas, como el caso del virus ISA $\mathrm{u}$ otras patologías, que generan un enorme impacto económico y social, tiene un componente ambiental relevante que no debe ser ignorado (Mardones et al. 2009). No se puede paliar el problema con soluciones exclusivamente veterinarias y aplicación de fármacos, sin definir las variables ambientales que están influyendo. En consecuencia, la solución requiere información científica independiente que permita identificar, en forma clara y transparente, tanto los factores inductores y de transferencia de patógenos (naturales y artificiales) como los problemas ambientales existentes. La situación requiere desarrollar estrategias productivas que tiendan a disminuir las consecuencias ambientales del monocultivo de especies animales y la producción de nitrógeno inorgánico con efectos de eutrofización costera.

Chile ha realizado esfuerzos importantes para generar una legislación efectiva frente a los problemas medioambientales más relevantes. Sin embargo es necesario preguntarse cómo se sustenta esta reglamentación y si tiende a innovar modelos productivos que pretendan alcanzar con efectividad una mayor sustentabilidad ambiental. Esta reglamentación (RAMA) se basa principalmente en el seguimiento del estado de los sedimentos bajo los sistema de cultivo, utilizando como indicador la detección de anaerobiosis (ausencia de oxígeno en normativa original) o baja concentración de oxígeno (normativa actual) como señal que genera procedimientos de contingencia (ver Buschmann et al. 2006b). Se han realizado esfuerzos para mantener una reglamentación actualizada y ésta ha sufrido varias modificaciones importantes desde el 2005 a la fecha (ver Tabla 1). Sin embargo, volvemos a la pregunta inicial, ¿en qué criterios está basada esta reglamentación? Nos parece que estos temas requieren una atención explícita, tanto del sector gubernamental como del sector productivo. Es evidente que la detección de condición anóxica no es suficiente indicador de la sustentabilidad ambiental, cuestiona la calidad de la reglamentación vigente y no promueve, en forma significativa, la búsqueda de soluciones y alternativas tecnológicas que otorguen mayor grado de sustentabilidad a esta actividad (Buschmann et al. 2006b). En la actualidad existen soluciones tecnológicas para abordar estas situaciones (ver a modo de ejemplo Buschmann et al. 2008c), sin embargo, requieren previamente identificar y definir, clara y transparentemente, los problemas ambientales, asignando a cada cual soluciones concretas y factibles. De este modo Chile podrá integrarse coherentemente en un mercado global ambientalmente cada vez más exigente. En la siguiente sección pretendemos establecer las restricciones y desafíos existentes para el desarrollo de una acuicultura más sustentable en Chile, evaluando la relevancia del uso de algas para alcanzar este propósito.

\section{OPORTUNIDADES Y RESTRICCIONES PARA EL CULTIVO DE ALGAS EN CHILE}

Si bien los mercados de ficocoloides siguen expandiéndose a nivel global, su expansión durante la última década fue mucho menor que hace 20 años (Bixler \& Porse 2011). Considerando la competencia por nuevos mercados y la capacidad de China y otros países orientales, de producir biomasa a bajo precio, generan una limitación para la expansión del cultivo de algas en Chile. Solo el descubrimiento de nuevas aplicaciones que le aporten mayor valor agregado a los ficocoloides, como alginatos en la producción de baterías de mayor eficiencia (Kovalenko et al. 2011) u otros usos innovadores, podrían incrementar la necesidad del cultivo de algas con mayor demanda y mejores precios. Además del tradicional uso como fuente de ficocoloides, la actual demanda de macroalgas como Macrocystis, para insumo en ciertas dietas animales, como la de abalón (FloresAguilar et al. 2007), ha generado un aumento de las restricciones de extracción de ciertas especies en Chile (Vásquez 2008). Además, el uso de biomasa para la producción de biocombustibles, se está transformando en una nueva alternativa a nivel global (e.g., Chisti 2008, Wargacki et al. 2012), por lo que es necesario desarrollar la tecnología que permita un cultivo industrializado cuyo costo 


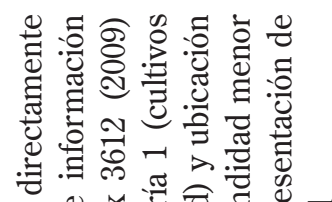

\% 若 $\|$ o 40

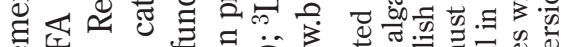

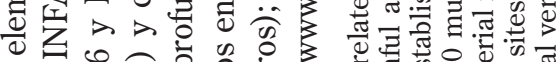
o

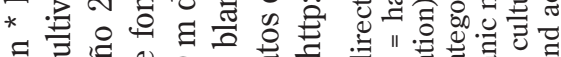
ठี

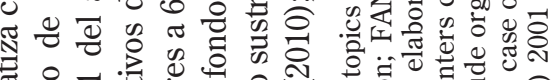

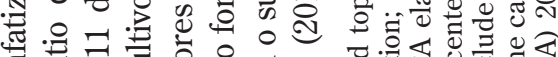

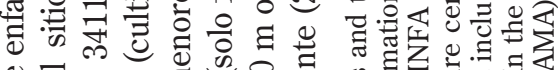
\&

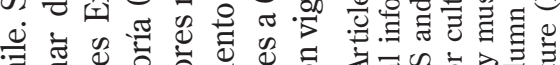

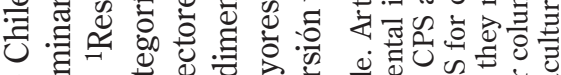

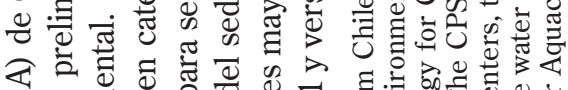

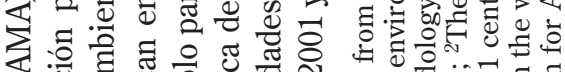

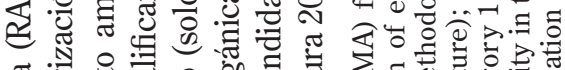
눈 윤

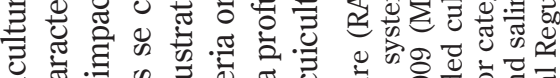

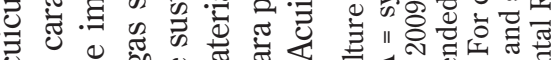

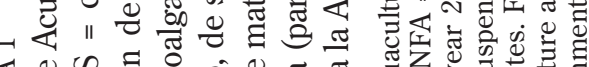

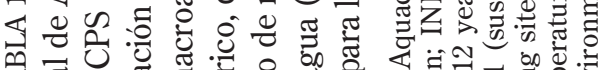

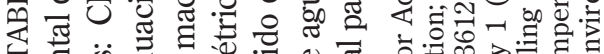

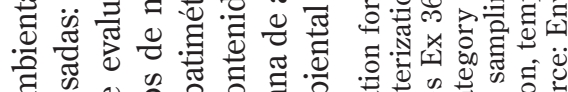

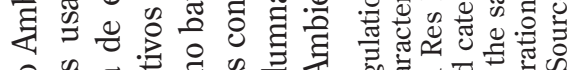

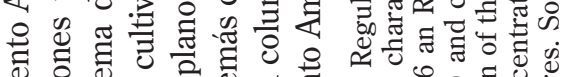

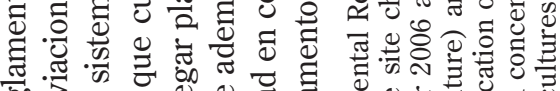

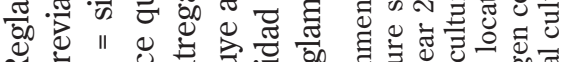

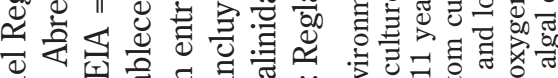

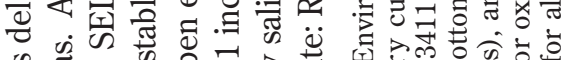

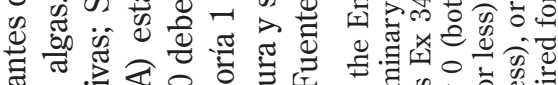

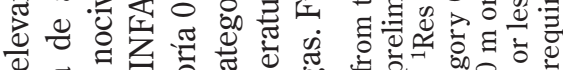
ए

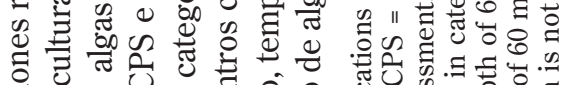

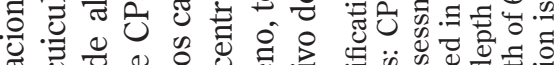

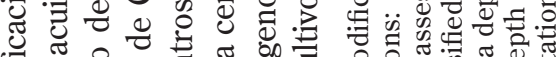

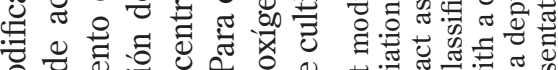
。

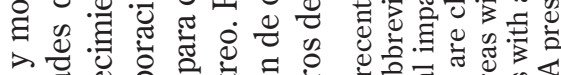

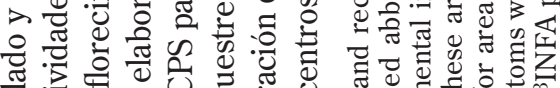

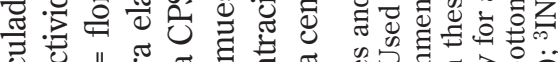

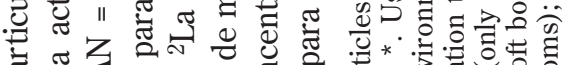

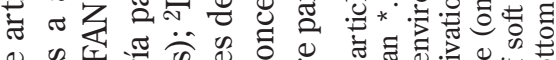

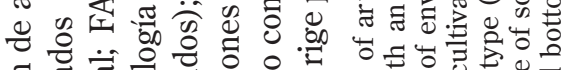

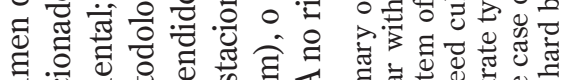

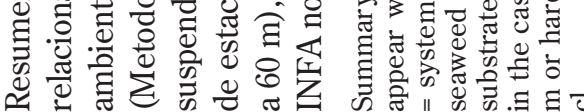

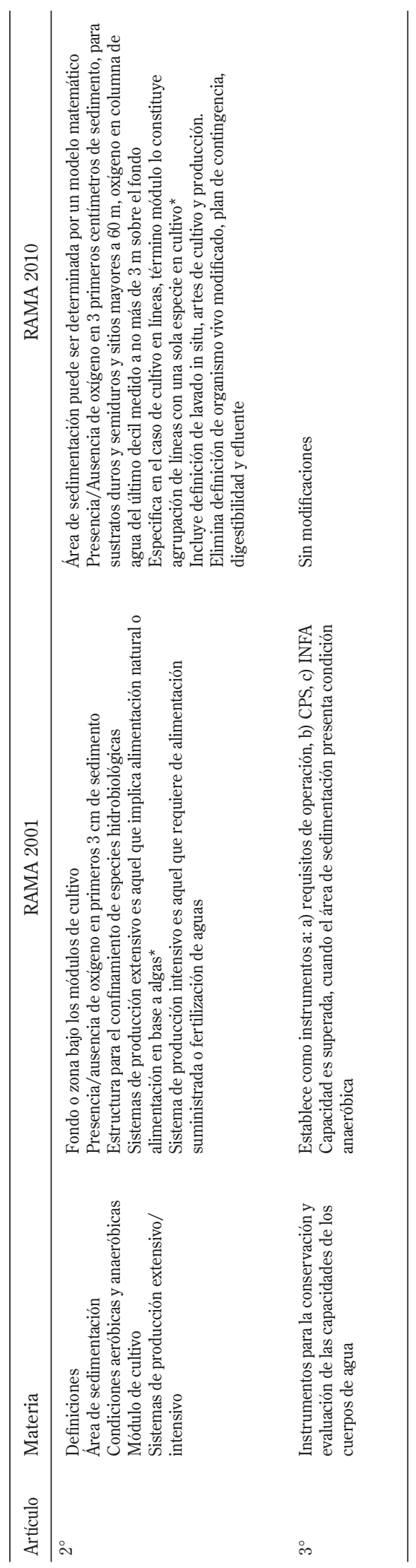




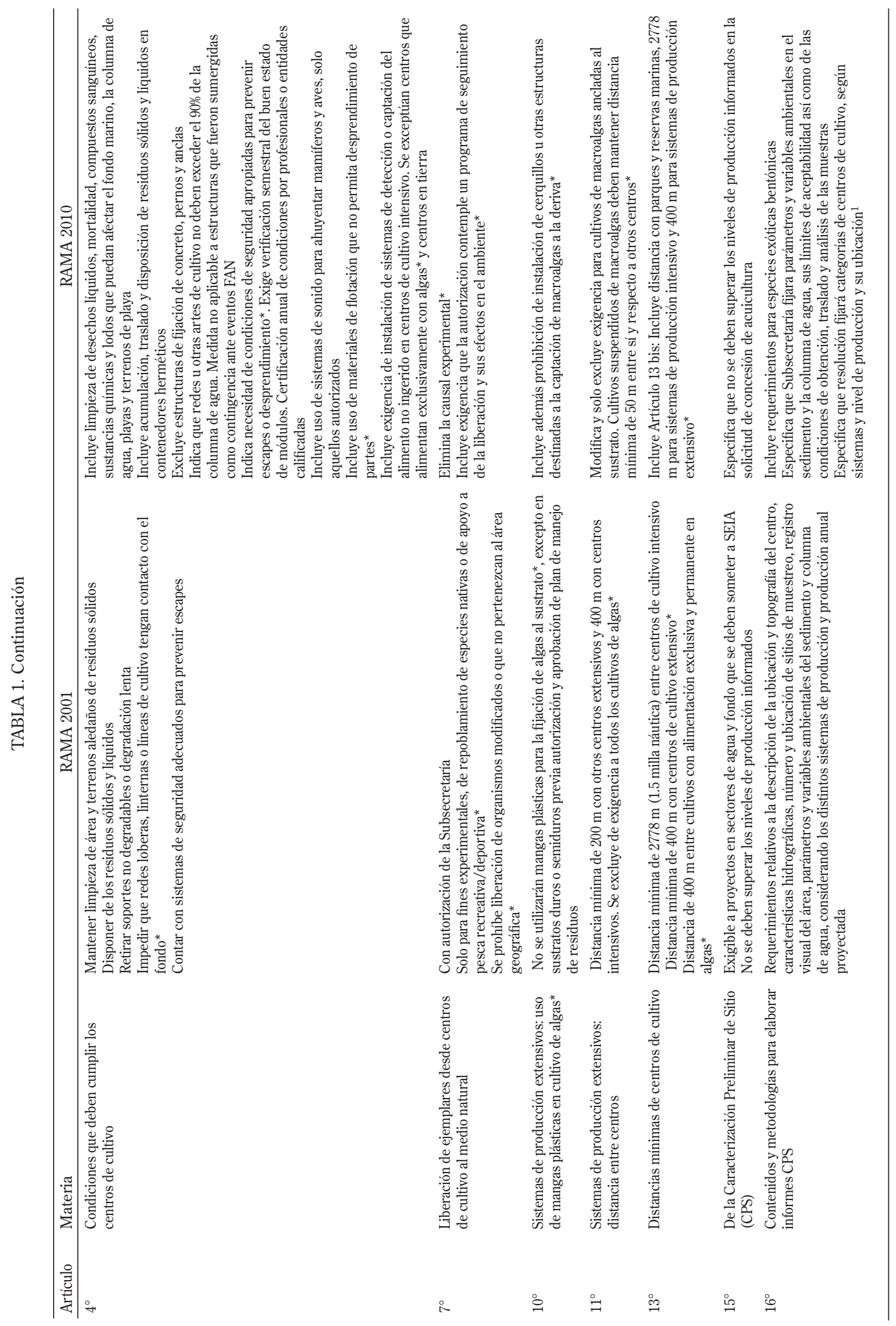




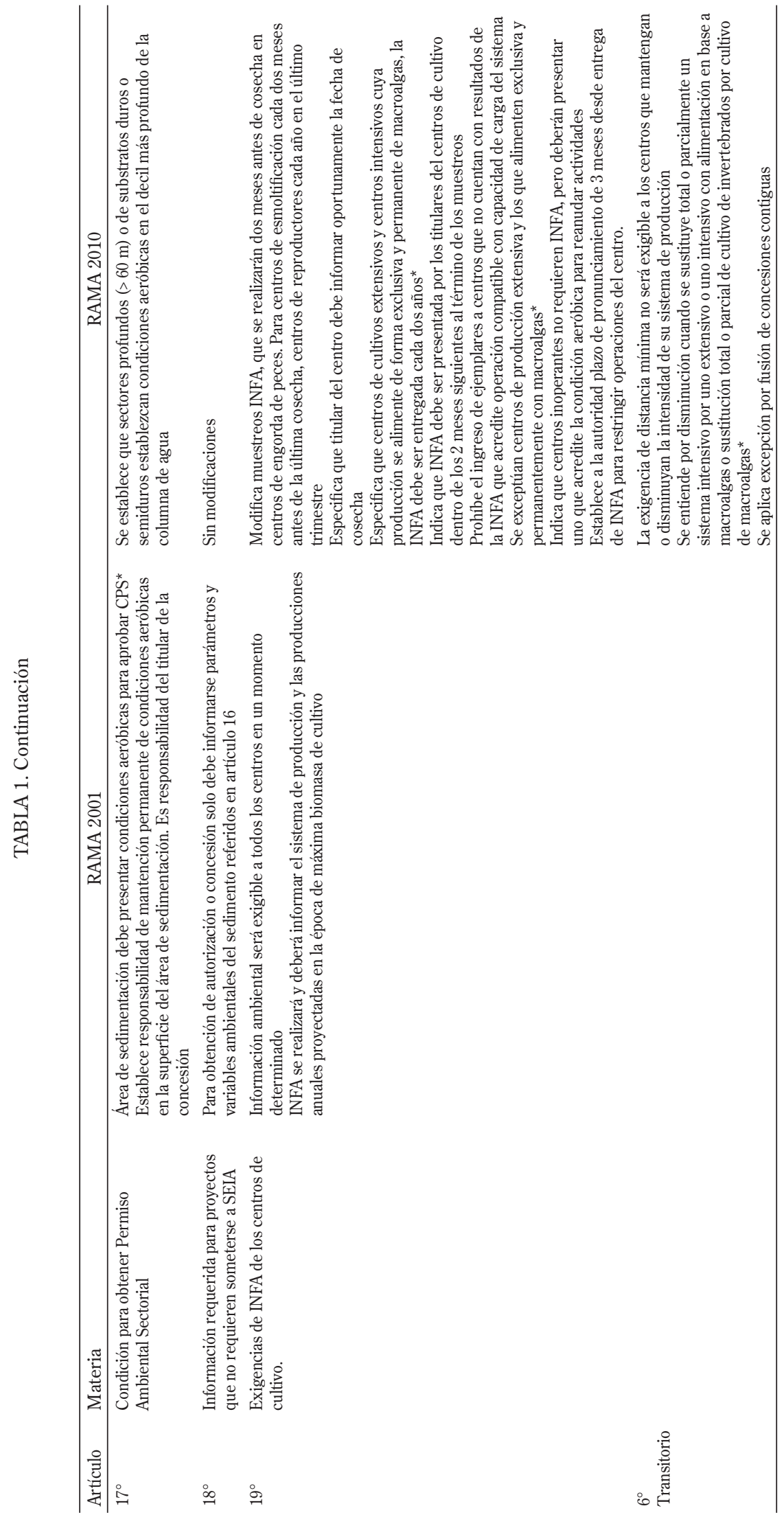


sea competitivo con los precios de mercado y con otras fuentes de biocombustibles (Maceiras et al. 2011). Se debe alcanzar niveles de eficiencia energética (Walker 2009, Clarens et al. 2010, Marcquardt 2011) que permitan que el desarrollo del cultivo de algas no solo sea económicamente rentable, sino que además tenga externalidades ambientales positivas. Los trabajos citados en este párrafo muestran cómo están emergiendo nuevos usos para las algas, implicando nuevas demandas de biomasa. Esta situación exige mejorar la rentabilidad de las prácticas de acuicultura (Buschmann et al. 2008b).

El otro polo de desarrollo del cultivo de algas, que hoy se conoce como Acuicultura integrada Multi-Trófica (IMTA), está asociado a su uso como biorremediador en procesos de eutrofización de zonas costeras donde se realizan actividades de acuicultura (Chopin et al. 2008, Buschmann et al. 2008a). Podemos señalar que un cultivo de algas es capaz de reducir un $85 \%$ de las emisiones de nitrógeno liberadas al medio en un ciclo anual de producción de salmones, reconociendo que este nutriente es el elemento de mayor impacto en los procesos de eutrofización costera (Buschmann et al. 1996a). En Chile se han realizado diversos estudios sobre IMTA, probando la capacidad de ciertas algas rojas $\mathrm{y}$ pardas para remover nitrógeno y fósforo inorgánico (Troell et al. 1997, Buschmann et al. 2008c, Abreu et al. 2009). La eficiencia de retiro de materia inorgánica disuelta requiere, en términos relativos, una gran superficie de algas con respecto a la superficie usada para el cultivo de peces (se requieren al menos 100 hectáreas de Macrocystis para remover un 80\% de los aportes de nitrógeno disuelto producido por una hectárea destinada al cultivo de peces, Buschmann et al. 2008c). Esta relación se basa en que mientras el cultivo de peces ocupa un volumen determinado por jaulas que alcanzan profundidades de 10 o más metros, en el caso de las algas, la productividad depende de la radiación solar, por lo que el uso de la columna de agua es limitado (Abreu et al. 2009). Por ello, para que el cultivo de algas se transforme en una alternativa ambiental económicamente sustentable se necesita generar un marco normativo que permita valorizar los procesos de biorremediación (Chopin et al. 2001, Neori et al. 2007). La valorización ambiental del cultivo de algas puede realizarse por medio de la internalización de los costos ambientales (Buschmann et al. 1996b) o por la creación de incentivos al cultivo (Buschmann et al. 2008a).

Se puede concluir que, aunque algunas actividades tradicionales tienen menor vigor económico que antaño, han emergido nuevas alternativas productivas (ver párrafos anteriores) que debiesen potenciar el desarrollo del cultivo de las algas en Chile y en otros países occidentales. En Chile, pese a existir tecnologías de producción para cierta variedad de algas, el desarrollo del cultivo de estas es escaso y poco diversificado, lo que parece asociado a una baja demanda y precios poco atractivos (Buschmann et al. 2008b, Bixler \& Porse 2011). Será difícil prosperar si no se instauran incentivos que fomenten cambios en la estructura de producción acuícola o incentivos económicos para reducir las cargas de nutrientes disueltos en el agua a través del uso de macroalgas. Por ahora, solo se aprecia como demandante de biomasa significativo, el potencial que presentan ciertas especies, especialmente Macrocystis, para ser utilizada para la producción de bioetanol (Wargacki et al. 2012).

El Reglamento Ambiental de Acuicultura (RAMA) regula parte de las actividades de acuicultura en Chile (Tabla 1). Este se aprobó en el año 2001 en el contexto de la Ley General de Pesca y Acuicultura (LGPA) (Promulgada en septiembre 1991 y publicada en enero 1992). Esta normativa dispone la necesidad de reglamentar las medidas de protección del medio ambiente para que los establecimientos de acuicultura operen en niveles compatibles con las capacidades de los cuerpos de agua, y responsabiliza al concesionario de las alteraciones en la limpieza y el equilibrio ecológico, producto de las actividades acuícolas en la zona concesionada (Artículos 84 y 87, LGPA). Desde su aprobación en el año 2001, este reglamento ha sido modificado, a partir del año 2005, por cinco resoluciones, principalmente con la intención de adecuar la letra de la norma a su aplicación práctica, incluyendo mayor especificación de términos, responsabilidades y restricciones (Tabla 1). Gran parte del RAMA está dedicado a establecer los criterios que definen el concepto de "daño ambiental" y las metodologías para la caracterización preliminar del sitio de 
cultivo (CPS) y de información ambiental (INFA). Estas metodologías están destinadas a evaluar tanto al inicio, como a monitorear en el tiempo, los indicadores ambientales de la capacidad (de carga) del cuerpo de agua sujeto a actividad acuícola, indicando si esta ha sido superada o no. Este monitoreo permanente permite establecer en qué momento el área de sedimentación de las instalaciones productivas presentan condiciones anaeróbicas, lo que equivale a que ciertos valores en las variables del sedimento o de la columna de agua han sido sobrepasadas (i.e. $\mathrm{O}_{2} \leq 2,4 \mathrm{mg} \mathrm{L}^{-1}$, contenido de materia orgánica $\geq 9,1 \%, \mathrm{pH} \leq 6,7$, y ausencia de cubiertas de microorganismos visibles y de burbujas de gas) (Res. Ex. 2411 2009). Mediante la categorización de los centros de cultivo, el instrumento legal reconoce diferencias en el grado de impacto generado por diferentes actividades acuícolas de acuerdo a la biomasa en producción y si utilizan nutrientes y alimento natural (sistema extensivo) o requieren de suministro externo de alimento o fertilizantes (sistema intensivo). Sin embargo, la normativa actual no explicita el rol del aporte de nutrientes en la degradación ambiental y su efecto en la disminución de la capacidad de carga. El protagonismo de las macroalgas en la remoción de nutrientes está reconocido solo en forma implícita por el RAMA ya que considera que generan un impacto menor, no estableciendo restricción de distancia con otros centros de cultivo. Por otra parte solo se le exige la presentación de un informe de CPS (Caracterización Preliminar de Sitio) que incluye la medición del mínimo de variables ambientales (caracterización del sustrato y materia orgánica del sedimento) en la solicitud de concesión, y la exime de presentar INFA (Información Ambiental), asumiendo que el cultivo de algas no contribuye a generar sedimentación significativa de partículas que favorezcan condiciones anaeróbicas del sustrato. Sin embargo, grandes extensiones de moluscos y/o macroalgas en sistemas suspendidos, o anclados al fondo, pueden reducir la velocidad de la corriente y con ello aumentar la tasa de sedimentación (e.g. Buschmann et al. 1997, McKindsey et al. 2011). La normativa debiese contemplar mediciones e indicadores apropiados con el cultivo masivo de macroalgas, considerando tanto la especie, el tipo de sistema de cultivo, como la biomasa producida y su extensión de cultivo. Los objetivos de las últimas modificaciones a la norma original no están orientados a beneficiar la capacidad de carga de los cuerpos de agua utilizados para cultivo, o a maximizar la capacidad de absorber el exceso de nutrientes producidos por actividades emisoras como son la acuicultura intensiva, las ciudades o el escurrimiento de terrenos agrícolas fertilizados.

Por otra parte, la acuicultura de algas debe realizarse en condiciones que minimice su impacto ambiental. En este sentido, la normativa actual impide el uso de mangas de polietileno en sustratos blandos, aunque sí lo permite en sustratos duros y semiduros cuando haya sido aprobado por la autoridad y siempre que se contemple un plan de manejo de estos residuos. Actualmente se prohíbe la utilización de sistemas de flotación que puedan disgregarse (e.g., poliestireno expandido) para evitar su acumulación como basura en las playas del litoral. Claramente esta normativa tiene sesgos asociados a que solo Gracilaria presenta cultivos a escala comercial. Sin embargo, y como se ha comentado con anterioridad, se augura el desarrollo del cultivo de otras especies que utilizan otros sistemas de producción como es el caso de Macrocystis donde los protocolos de acuicultura apuntan hacia sistemas suspendidos de producción (Gutiérrez et al. 2006, Westermeier et al. 2006, Macchiavello et al. 2010), aunque, tal y como se ha señalado, aún persisten situaciones de mercado que no han permitido su desarrollo. En consecuencia, parece necesario incorporar al RAMA y a las regulaciones de acuicultura en general, las especificidades del cultivo de algas, con toda su diversidad y requerimientos, con el fin de no generar distorsiones reglamentarias que inhiban el futuro desarrollo de la acuicultura de algas en Chile.

\section{CONCLUSIONES}

Chile tiene condiciones ambientales extremadamente favorables para realizar considerable desarrollo acuícola toda vez que los recursos pesqueros están siendo cada vez más escasos tanto a nivel nacional como mundial (Jackson et al. 2001, Pauly et al. 2002, Worm et al. 2009). Ello debe abrir nuevas oportunidades comerciales para el desarrollo de la acuicultura (Duarte et al. 2007). 
Sin embargo, esta oportunidad de negocio no puede sustentarse en el menoscabo del patrimonio ambiental del país y deben buscarse fórmulas y estrategias que permitan alcanzar metas productivas donde el componente ambiental esté descrito en forma explícita. La diversificación acuícola debe estar basada en la incorporación de especies con funciones ecológicas diferentes (productores primarios, detritívoros, herbívoros y carnívoros), con el fin de balancear el flujo de materia y energía en sistemas costeros utilizados por prácticas de acuicultura. Por ello, la generación de información científica es un elemento imprescindible para la sustentabilidad de la acuicultura en Chile (Buschmann et al. 2009, Medina 2009) y es necesario implementar las propuestas tecnológicas e innovaciones existentes que permitan resolver las deficiencias anteriormente descritas. En particular, no pueden estar ausentes los servicios ecosistémicos que aportan las algas, con el fin de balancear flujos de materia y energía. Se necesitan sistemas de incentivo que permitan fomentar esta actividad y así avanzar hacia un uso racional de nuestros sistemas costeros. La eficiencia ecológica, la sustentabilidad ambiental y la rentabilidad económica del cultivo de organismos carnívoros puede ser mejorada cuando esta actividad se realiza en un sistema ecológicamente balanceado. Esto requiere, desde una perspectiva de acuicultura integrada multitrófica, integrar especies de bajo nivel trófico (Ridler et al. 2007, Neori \& Nobre 2012) y además un contexto regulatorio más apropiado. Es necesario internalizar costos ambientales del uso costero con el fin de incentivar la aplicación de tecnologías (IMTA) que disminuyan las externalidades negativas y los costos asociados (Buschmann et al. 1996b, 2008a, Chopin et al. 2001). Además es necesario generar valor agregado a las algas, potenciando así incrementar su demanda. Si Chile aborda este tema en profundidad, no solo podría ser un exportador de productos alimentarios, sino además de tecnologías de producción sustentable para la acuicultura mundial.

AGRADECIMIENTOS: El presente manuscrito fue preparado y presentado en Simposio de Algas realizado en el marco del Congreso de Ciencias del Mar 2011. AHB agradece la invitación por parte de la directiva de la Sociedad de Ficología de Chile. Este trabajo está basado en experiencias llevadas a cabo durante los últimos 20 años, apoyados principalmente por FONDECYT. Los autores agradecen la crítica de ambos evaluadores que permitieron mejorar este trabajo sustancialmente.

\section{LITERATURA CITADA}

ABREU MH, DA VARELA, L HENRÍQUEZ, A VILLARROEL, C YARISH, I SOUSA-PINTO \& AH BUSCHMANN (2009) Traditional vs. integrated multi-trophic aquaculture of Gracilaria chilensis C J Bird, J McLachlan \& EC Oliveira: productivity and physiological performance. Aquaculture 293: 211-220.

ANDERSON DM, GLIBERT PM \& JM BURKHOLDER (2002) Harmful algal blooms and eutrophication: Nutrient sources, composition, and consequences. Estuaries 25: 704-726.

BECKER LA, MA PASCUAL \& NG BASSO (2007) Colonization of the southern Patagonia ocean by exotic chinook salmon. Conservation Biology 21: 1347-1352.

BIXLER HJ \& H PORSE (2011) A decade of change in seaweed hydrocolloids industry. Journal of Applied Phycology 23: 321-335.

BRAVO S \& PJ MIDTLYNG (2007) The use of fish vaccines in the Chilean salmon industry 19992003. Aquaculture 270: 36-42.

BUSCHMANN AH \& R PIZARRO (2001) El costo ambiental de la salmonicultura en Chile. Análisis de Políticas Públicas, Fundación Terram (Chile) 5: $1-7$.

BUSCHMANN AH \& A FORTT (2005) Efectos ambientales de la acuicultura intensiva y alternativas para un desarrollo sustentable. Ambiente y Desarrollo (Chile) 21: 58-64.

BUSCHMANN AH, M TROELL, N KAUTSKY \& L KAUTSKY (1996a) Integrated tank cultivation of salmonids and Gracilaria chilensis (Rhodophyta). Hydrobiologia 326/327: 75-82.

BUSCHMANN AH, DA LÓPEZ \& A MEDINA (1996b) A review of the environmental effects and alternative production strategies of marine aquaculture in Chile. Aquacultural Engineering 15: 397-421.

BUSCHMANN AH, F BRIGANTI, \& CA RETAMALES (1997) Intertidal cultivation of Gracilaria chilensis (Rhodophyta) in southern Chile: long term invertebrate abundance patterns. Aquaculture 156: 269-278.

BUSCHMANN AH, JA CORREA, R WESTERMEIER, MC HERNÁNDEZ-GONZÁLEZ \& R NORAMBUENA (2001) Red algal farming in Chile: a review. Aquaculture 194: 203-220.

BUSCHMANN AH, VA RIQUELME, MC HERNÁNDEZGONZÁLEZ, DA VARELA, D, JE JIMÉNEZ et al. (2006a) A review of the impacts of salmon farming on marine coastal ecosystems in the southeast Pacific. ICES Journal of Marine Science 63:13381345.

BUSCHMANN AH, VA RIQUELME, MC HERNÁNDEZ-GONZÁLEZ \& LA HENRÍQUEZ (2006b) Additional perspectives for ecosystem approaches for aquaculture. In: McVey JP, C-S Lee \& PJ O’Bryan (eds) Aquaculture and ecosystems: An integrated coastal and ocean management approach: 168-176. The World Aquaculture Society, Baton Rouge, Louisiana, US. 
BUSCHMANN AH, MC HERNÁNDEZ-GONZÁLEZ, C ARANDA, T CHOPIN, A NEORI, C HALLING \& M TROELL (2008a) Mariculture waste management. In: Jorgensen SE \& BD Fath (eds) Encyclopedia of Ecology, Ecological Engineering, Vol 3: 2211-2217. Elsevier, Oxford, Great Britain.

BUSCHMANN AH, MC HERNÁNDEZ-GONZÁLEZ \& DA VARELA (2008b) Seaweed future cultivation in Chile: perspectives and challenges. International Journal of Environment and Pollution 33: 432-456.

BUSCHMANN AH, DA VARELA, MC HERNANDEZGONZÁLEZ \& P HUOVINEN (2008c) Opportunities and challenges for the development of an integrated seaweed-based aquaculture activity in Chile: Determining the physiological capabilities of Macrocystis and Gracilaria as biofilters. Journal of Applied Phycology 20: 571-577.

BUSCHMANN AH, F CABELLO, K. YOUNG, J CARVAJAL, DA VARELA, \& LA HENRÍQUEZ (2009) Salmon aquaculture and coastal ecosystem health in Chile: Analysis of regulations, environmental impacts and bioremediation systems. Ocean \& Coastal Management 52: 243-249.

BUSCHMANN AH, A TOMOVA, A LÓPEZ, MA MALDONADO, LA HENRÍQUEZ, L IVANOVA, F MOY, HP GODFREY \& FC CABELLO (2012) Salmon aquaculture and antimicrobial resistance in the marine environment. PlosOne 7: e42724. doi:10.1371/journal.pone.0042724

BUSTOS, PA, ND YOUNG, MA ROZAS, HM BOHLE, RS ILDEFONSO, RN MORRISON \& BF NOWAK (2011) Amoebic gill disease (AGD) in Atlantic salmon (Salmo salar) farmed in Chile. Aquaculture 110: 281-288.

CABELLO FC (2006) Heavy use of prophylactic antibiotics in aquaculture: a growing problem for human and animal health and for the environment. Environmental Microbiology 8: 1137-1144.

CARVAJAL J, L GONZÁLEZ \& M GEORGENASCIMENTO (1998) Native sea lice infestation of salmonids reared in netpen system in southern Chile. Aquaculture 166: 241-246.

CHELOSSI E, L VEZZULLI, A MILANO, M BRANZONI, M FABIANO, G RICCARDI, IM BANAT (2003) Antibiotic resistance of benthic bacteria in fishfarm and control sediments of the Western Mediterranean. Aquaculture 219: 83-97.

CHISTI Y (2008) Biodiesel from microalgae. Biotechnology Advances 25: 294-306.

CHOPIN T, AH BUSCHMANN, C HALLING, M TROELL, N KAUTSKY, et al. (2001) Integrating seaweeds into marine aquaculture systems: a key toward sustainability. Journal of Phycology 37: 975-986.

CHOPIN T, SMC ROBINSON, M TROELL, A NEORI, AH BUSCHMANN \& J FANG (2008) Multitrophic integration for sustainable marine aquaculture. In: Jørgensen SE \& BD Fath (eds) Encyclopedia of Ecology, Ecological Engineering, Vol 3: 2463-2475. Elsevier, Oxford, Great Britain.

CHOPIN T, A NEORI, A BUSCHMANN, S PANG \& M SAWHNEY (2011) Diversification of the aquaculture sector: Seaweed cultivation, integrated multi-trophic aquaculture, integrated sequential biorefineries. Global Aquaculture Advocate (USA): 58-60.

CLARENS AF, EP RESURRECCION, MA WHITE \& LM COLOSI (2010) Environmental life cycle comparison of algae to other bioenergy feedstocks. Environmental Science Technology 44: 1813-1819.

CLARKE AL, K WECKSTRÖM, DJ CONLEY, NJ ANDERSON, F ADSER et al. (2006) Long-term trends in eutrophication and nutrients in the coastal zone. Limnology and Oceanography 51: 385-397.

CONLEY DJ, HW PAERL, RW HOWARTH, DF BOESCH, SP SEITZINGER, KE HAVENS, C LANCELOT \& GE LIKENS (2009) Controlling eutrophication: nitrogen and phosphorus. Science 323: 1014-1015.

DUARTE CM, N MARBÁ \& M HOLMER (2007) Rapid domestication of marine species. Science 316 : 382-383.

FAO (2010) Statistics, FAO Fisheries and Aquaculture Department, Rome, Italy. http://www.fao.org/ fishery/statistics (accedido Marzo 2012).

FLORES-AGUILAR RA, GUTIÉRREZ A, ELLWANGER A, SEARCY-BERNAL R (2007) Development and current status of abalone aquaculture in Chile. Journal of Shellfish Research 26: 705-711.

FOLKE C \& KAUTSKY N (1989) The role of ecosystems for a sustainable development of Aquaculture. Ambio 18: 234-243.

FOLKE C, N KAUTSKY, H BERG, A JANSSSON \& M TROELL (1998) The ecological footprint concept for sustainable seafood production: a review. Ecological Applications 8: S63-S71.

FORTT A, F CABELLO \& AH BUSCHMANN (2007) Residuos de tetraciclina y quinolonas en peces silvestres en una zona costera donde se desarrolla la acuicultura del salmón en Chile. Revista Chilena de Infectiología 24: 8-12.

GODOY MC, A AEDO, MJT KIBENGE, DB GROMA, CV YASON et al. (2008) First detection, isolation and molecular characterization of infectious salmon anaemia virus associated with clinical disease in farmed Atlantic salmon (Salmo salar) in Chile. BMC Veterinary Research 4: doi:10.1186/1746-6148-4-28.

GUTIÉRREZ A, CORREA T, MUÑOZ V, SANTIBAÑEZ A, MARCOS R, CÁCERES C \& BUSCHMANN AH (2006) Farming of the giant kelp Macrocystis pyrifera in southern Chile for development of novel food products. Journal of Applied Phycology 18: 259-267

HARGRAVE BT (2010) Empirical relationships describing benthic impacts of salmon aquaculture. Aquculture Environment Interactions 1: 33-46.

HAYA K, LE BURRIDGE, \& BD CHANG (2001) Environmental impact of chemical wastes produced by the salmon aquaculture industry. ICES Journal of Marine Science 58: 492-496.

HEUER OE, H KRUSE, K GRAVE, P COLLIGNON, I KARUNASAGAR \& FJ ANGULO (2009) Human health consequences of use of antimicrobial agents in aquaculture. Food Safety 49: 1248-1253.

JACKSON JBC, MX KIRBY, WH BERGER, KA BJORNDAL, LW BOTSFORD, et al. (2001) Historical overfishing and the recent collapse of coastal ecosystems. Science 293: 629-637.

KOVALENKO I, B ZDYRKO, A MAGASINSKI, B HERTZBERG \& Z MILICEV (2011) A major constituent of brown algae for use in high-capacity Li-ion. Science 334: 75-79.

LIU F, S PANG, T CHOPIN, S GAO, T SHAN, $\mathrm{X}$ ZHAO \& J LI (2013) Understanding the recurrent large-scale green tide in the Yellow Sea: Temporal and spatial correlations between 
multiple geographical, aquacultural and biological factors. Marine Environmental Research 83: 38-47

MACCHIAVELLO J, E ARAYA \& C BULBOA (2010) Production of Macrocystis pyrifera (Laminareales; Phaeophyceae) in Northern Chile on spore-based culture. Journal of Applied Phycology 22: 691-697.

MACEIRAS R, M RODRÍGUEZ, A CANCELA, S URREJOLA S, \& A SÁNCHEZ (2011) Macroalgae: Raw material for biodiesel production. Applied Energy 88: 3318-3323.

MARDONES FO, AM PEREZ, TE CARPENTER (2009) Epidemiologic investigation of the re-emergence of infectious salmon anemia virus in Chile. Disease of Aquatic Organisms 84: 105-114.

MARQUARDT R (2011) Light acclimatation of aquatic photoautotrophs -applied aspects. Tesis de Doctorado, Universität Rostock, Alemania.

MCKINDSEY CW, P ARCHAMBAULT, MD CALLIER, $\&$ F OLIVIER (2011) Influence of suspended and off-bottom mussel culture on the sea bottom and benthic habitats: a review. Canadian Journal of Zoology 89: 622-646

MEDINA MH (2009) En salmonicultura, la I+D retoma su camino. Aqua (marzo) (Chile): 122-125.

NAYLOR RL, RJ GOLDBURG, JH PRIMAVER, N KAUTSKY, MCM BEVERIDGE, J CLAY, C FOLKE, J LUBCHENCO, H MOONEY \& M TROELL (2001) Effect of aquaculture on world fish supplies. Nature 405: 1017-1024.

NEORI A \& AM NORBE (2012) Relationship between trophic level and economics in aquaculture. Aquaculture Economics \& Management, 16: 40-67

NEORI A, M TROELL, T CHOPIN, C YARISH, A CRITCHLEY \& AH BUSCHMANN (2007) The need for a balanced ecosystem approach to blue revolution aquaculture. Environment 49: 37-44.

NONAKA L, K IKENO \& S SUZUKI (2007) Distribution of tetracycline resistance gene, tet $(\mathrm{M})$, in Grampositive and Gram-negative bacteria isolated from sediment and seawater at a coastal aquaculture site in Japan. Microbes and Environments 22: 355-364.

OLSEN Y (2011) Resources for fish feed in future mariculture. Aquaculture Environmental Interaction 1: 187-200.

PAULY D, V CHRISTENSEN, S GUÉNETTE, TJ PITCHER, UR SUMAILA, CJ WALTERS, R WATSON \& D ZELLER (2002) Towards sustainability in world fisheries. Nature 418: 689-695.

PECKOL P \& JS RIVERS (1996) Contribution by macroalgal mats to primary production of a shallow embayment under high and low nitrogenloading rates. Estuarine Coastal and Shelf Science 43: 311-325.

RIDLER N, M WOWCHUK, B ROBINSON, K BARRINGTON, T CHOPIN, S ROBINSON, F PAGE, G REID, M SZEMERDA, J SEWUSTER, \& S BOYNE-TRAVIS (2007). Integrated multi trophic aquaculture (IMTA): a potential strategic choice for farmers. Aquaculture Economics and Management 11: 99-110.

SANTELICES B (1996) Seaweed research and utilization in Chile: moving into a new phase. Hydrobiologia 326/327: 1-14.

SEPÚLVEDA F, S MARIN \& J CARVAJAL (2004) Metazoan parasites in wild fish and farmed salmon from aquaculture sites in southern Chile. Aquaculture 235: 89-100.

SERNAPESCA (2012) Anuarios Estadística de Pesca, Servicio Nacional de Pesca y Acuicultura, Chile. http://www.sernapesca.cl/index. php?option $=$ com_remository $\&$ Itemid $=54 \&$ func $=$ se lect\&id=2 (accedido Marzo 2012).

SHI J, H WEI, L ZHAO, Y YUAN, J FANG \& J ZHANG (2011) A physical-biological coupled aquaculture model for a suspended aquaculture area of China. Aquaculture 318: 412-424.

SOTO D \& F NORAMBUENA (2004) Evaluation of salmon farming effects on marine systems in the inner seas of southern Chile: a large-scale mensurative experiment. Journal of Applied Ichthyology 20: 493-501.

SOTO D, F JARA \& CA MORENO (2001) Escaped salmon in the inner seas, southern Chile: Facing ecological and social conflicts. Ecological Applications 11: 1750-1762.

SOTO D, I ARISMENDI, J GONZALEZ, J SANZANA, F JARA, C JARA, E GUZMAN \& LARA A (2006) Southern Chile, trout and salmon country: invasion patterns and threats for native species. Revista Chilena Historia Natural 79: 97-117.

SOTO D, FJ SALAZAR \& MA ALFARO (2007) Considerations for comparative evaluation of environmental costs of livestock and salmon farming in southern Chile. In: Bartley DM, C Brugère, D Soto, P Gerber \& B Harvey (eds) Comparative assessment of the environmental costs of aquaculture and other food production sectors: methods for meaningful comparisons: 121-136. FAO, Rome, Italy.

TROELL M, C HALLING, A NILSSON, AH BUSCHMANN, N KAUTSKY \& L KAUTSKY (1997) Integrated open sea cultivation of Gracilaria chilensis (Gracilariales, Rhodophyta) and salmons for reduced environmental impact and increased economic output. Aquaculture156: 45-62.

TROELL M, P RÖNNBÄCK, C HALLING, N KAUTSKY \& AH BUSCHMANN (1999) Ecological engineering in aquaculture: use of seaweeds for removing nutrients from intensive mariculture. Journal of Applied Phycology 11: 89-97.

TROELL M, A JOYCE, T CHOPIN, A NEORI, AH BUSCHMANN \& J-G FANG (2009) Ecological engineering in aquaculture - Potential for integrated multi-trophic aquaculture (IMTA) in marine offshore systems. Aquaculture 297: 1-9.

VÁSQUEZ JA (2008) Production, use and fate of Chilean brown seaweeds: re-sources for a sustainable fishery. Journal of Applied Phycology 20: 457-467.

WALKER DA (2009) Biofuels, facts, fantasy and feasibility. Journal applied Phycology 21: 509-517.

WARGACKI AJ, E LEONARD, MN WIN, DD REGITSKY, CNS SANTOS, et al. (2012) An engineered microbial platform for direct biofuel production from brown macroalgae. Science 335: 308-313.

WESTERMEIER R, D PATIÑO, M PIEL, I MAIER, \& D MÜLLER (2006) A new approach to kelp mariculture in Chile: production of free-floating sporophyte seedlings from gametophyte cultures of Lessonia trabeculata and Macrocystis pyrifera. Aquaculture Research 37: 164-171.

WORM B, R HILBORN, JK BAUM, TA BRANCH, JS COLLIE, et al. (2009) Rebuilding global fisheries. Science 325: 578-585. 